\title{
The association of ulceration of the foot with cardiovascular and all-cause mortality in patients with diabetes: a meta-analysis
}

\author{
J. R. W. Brownrigg • J. Davey • P. J. Holt • W. A. Davis • \\ M. M. Thompson • K. K. Ray • R. J. Hinchliffe
}

Received: 2 April 2012 /Accepted: 6 July 2012 / Published online: 14 August 2012

(C) Springer-Verlag 2012

\begin{abstract}
Aims/hypothesis It is well established that diabetes mellitus increases the risk of cardiovascular disease (CVD) and allcause mortality. Observational studies suggest that a history of diabetic foot ulceration (DFU) may increase this risk further still. We sought to determine to what extent DFU is associated with excess risk over and above diabetes.

Methods We identified studies reporting on associations of DFU with CVD and all-cause mortality. We obtained data on incident events of all-cause mortality, fatal myocardial infarction and fatal stroke. Study-specific estimates were pooled using a random-effects meta-analysis and the statistical heterogeneity of included studies was assessed using the $I^{2}$ statistic. Results The eight studies included reported on 3,619 events of all-cause mortality during 81,116 person-years of followup. DFU was associated with an increased risk of all-cause mortality (RR 1.89, 95\% CI 1.60, 2.23), fatal myocardial infarction $(2.22,95 \%$ CI $1.09,4.53)$ and fatal stroke $(1.41$, 95\% CI $0.61,3.24)$. CVD mortality accounted for a similar proportion of deaths in DFU and non-DFU patients.
\end{abstract}

\footnotetext{
K. K. Ray and R. J. Hinchliffe contributed equally to this work.

J. R. W. Brownrigg · P. J. Holt • M. M. Thompson · R. J. Hinchliffe St George's Vascular Institute,

4th Floor, St James Wing, St George's Healthcare NHS Trust, Blackshaw Road,

London SW17 0QT, UK

J. Davey $\cdot$ K. K. Ray $\cdot$ R. J. Hinchliffe $(\square)$

Cardiac and Vascular Sciences, St George's University of London,

London, UK

e-mail: rhinchli@sgul.ac.uk

W. A. Davis

School of Medicine and Pharmacology,

University of Western Australia, Fremantle Hospital,

Fremantle, WA, Australia

Conclusions/interpretation Patients with DFU have an excess risk of all-cause mortality, compared with patients with diabetes without a history of DFU. This risk is attributable, in part, to a greater burden of CVD. If this result is validated in other studies, strategies should evaluate the role of further aggressive CVD risk modification and ulcer prevention in those with DFU.

Keywords Cardiovascular mortality · Diabetes · Diabetic foot ulcer $\cdot$ Meta-analysis $\cdot$ Mortality
Abbreviations
CAD Coronary artery disease
CAN Cardiovascular autonomic neuropathy
CVA Cerebrovascular accident
CVD Cardiovascular disease
DFU Diabetic foot ulceration
MI Myocardial infarction
PAD Peripheral arterial disease

\section{Introduction}

Cardiovascular disease (CVD) and all-cause mortality among individuals with diabetes mellitus remains approximately twofold higher compared with individuals without diabetes [1]. Several observational studies have suggested an even higher mortality rate in patients with diabetic foot ulceration (DFU), yet the size of this excess risk has not been well quantified. In addition, it remains unclear whether any excess mortality observed in patients with DFU reflects an increased burden of cardiovascular risk factors in patients or relates to potential sequelae of the ulcer. Importantly, increased duration of diabetes is also associated with greater 
risk of DFU [2], and strongly correlates with adverse outcomes, including CVD and all-cause mortality $[3,4]$.

Prior studies have been underpowered to provide reliable evidence of any potential impact of DFU on mortality. Such evidence would inform future strategies to assess whether prevention and/or better management of ulceration impact on CVD risk. More robust data regarding the relationship between DFU and mortality may justify a role for DFU in stratifying CVD risk among patients with diabetes and emphasise a need for aggressive treatment of CVD risk factors. We conducted a systematic review and meta-analysis of studies that reported information on both diabetes and DFU to assess more reliably than hitherto possible the extent to which DFU is associated with excess risk over and above diabetes.

\section{Methods}

Study selection We sought studies that reported on associations of DFU with all-cause mortality. We also sought, where available, cause-specific mortality data on fatal CVD outcomes (defined as fatal myocardial infarction [MI] and fatal cerebrovascular accident [CVA]). Our pre-specified inclusion criteria required studies with at least 1 year of follow-up that had simultaneously collected information on the presence or absence of DFU in a population of individuals with diabetes mellitus. Studies that selected only patients with lower-limb arterial-occlusive disease were excluded as these were unlikely to reflect more general diabetic populations. We searched electronic databases (PubMed, Embase, Cochrane Library) from their inception to August 2011 using both medical subject heading (MeSH) and keyword search terms; using 'diabetes', 'foot ulcer', 'cardiovascular', and 'mortality' retrieved 237 studies (Fig. 1). Following screening for the above inclusion criteria, the remaining studies were supplemented by

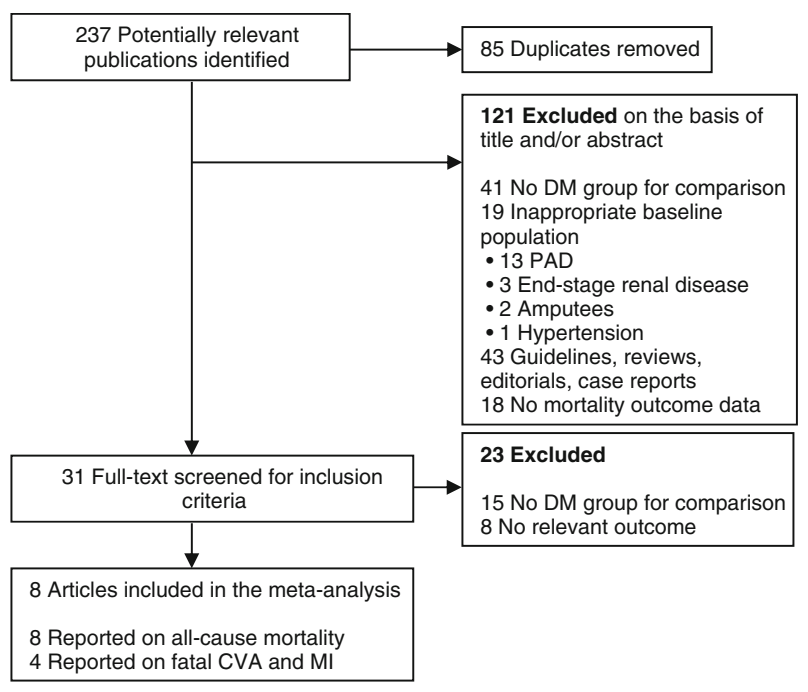

Fig. 1 Study selection. DM, diabetes only examining reference lists and by correspondence with study investigators to obtain additional unpublished data.

Information on the following variables was independently obtained by two investigators (JRWB and PJH) for both groups: study design; study size; various patient characteristics at baseline (age, time since diagnosis of diabetes, type of diabetes, the proportion of male patients; the presence of cardiovascular risk factors (hypertension, dyslipidaemia, coronary artery disease [CAD], cerebrovascular disease, smoking status); and the absolute number of events (fatal MI, fatal CVA, all-cause mortality). Any conflicts were resolved by discussion with a third investigator (RJH). Where studies reported on more than one occasion, the most recent data from the same cohort were used to reflect contemporary practice and to increase power.

Statistical analysis Investigators of the included studies were asked to provide missing data wherever possible. Summary characteristics of participants with and without DFU are presented as mean values weighted by study size. Differences in baseline characteristics between the groups were assessed using a combination of the $\chi^{2}$ test and unpaired $t$ tests for categorical and continuous data, respectively. We calculated the RRs and 95\% CIs for cardiovascular and allcause mortality based on observed data from individual studies. Study-specific estimates were pooled using a randomeffects meta-analysis, which accounts for heterogeneity in effect size among studies, using the method of DerSimonian and Laird [5]. Statistical heterogeneity of included studies was assessed using the $I^{2}$ statistic. The $I^{2}$ statistic is derived from Cochran's Q statistic and measures the proportion of overall variation in effect estimates that is attributable to betweenstudy heterogeneity. Crude event rates were calculated by dividing the absolute number of events by the total personyears of follow-up. To assess publication bias, we produced a funnel plot with its statistical analogue, the Begg-adjusted rank correlation test [6], and performed Egger's regression asymmetry test [7]. All statistical analyses were two-sided with a significance level of 0.05 ; calculations were performed with Stata release 11 (Stata corp, College Station, TX, USA).

\section{Results}

Eight studies published between 2006 and 2011 reporting on 17,830 patients and 81,116 person-years of follow-up were included (Fig. 1) [8-15]. Four studies were based in America, two in Europe, one in Australasia and one in South-East Asia. Individuals included were predominantly from clinic populations (Table 1). All studies reported included cohorts with both type 1 and type 2 diabetes, with the exception of one study [12], in which all participants had type 2 diabetes. The mean age in both groups was in the sixth decade, with a longer mean 


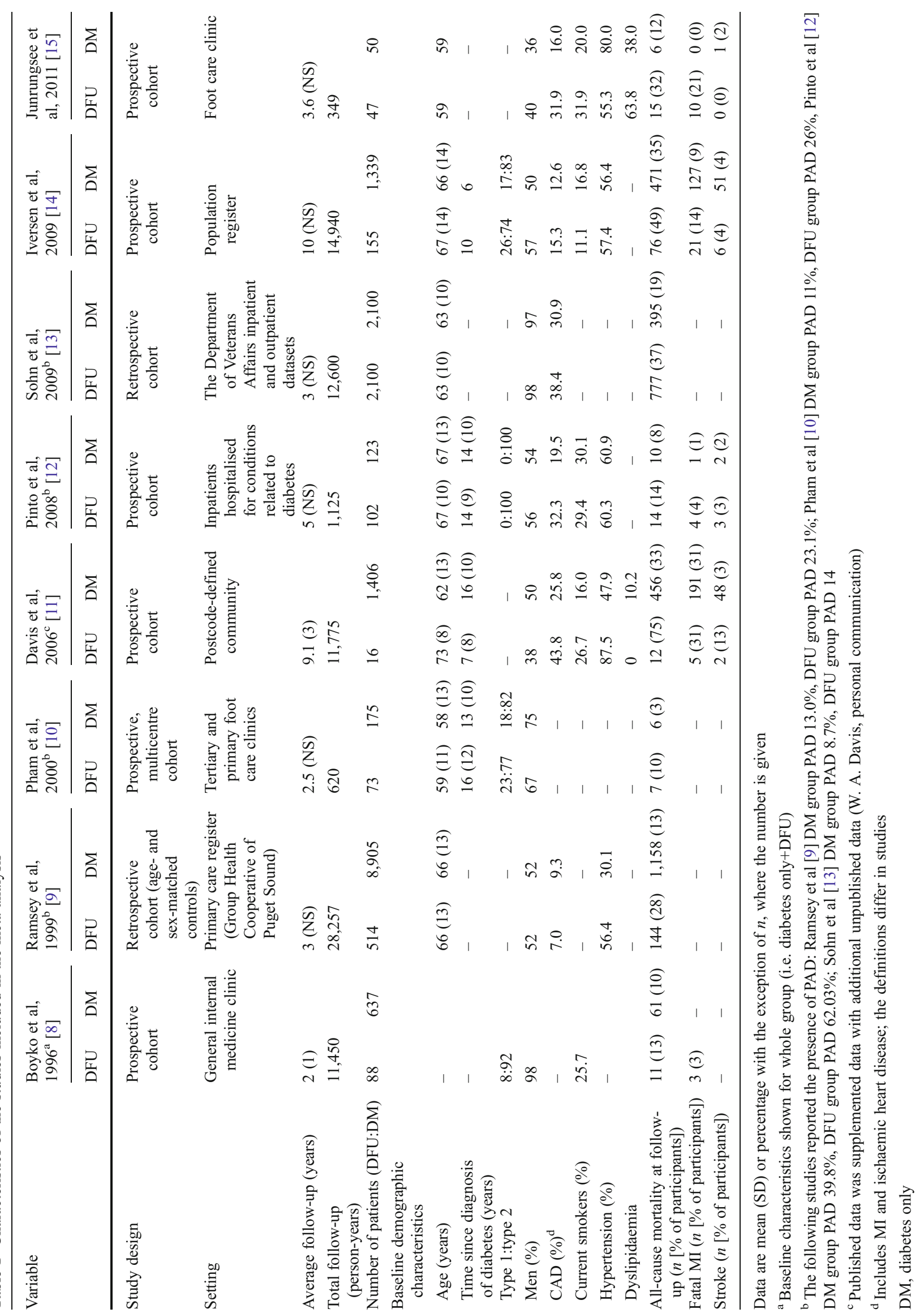


duration of diabetes in the DFU group (12.72 vs 7.19 years, $p<0.005)$. The prevalence of CAD, cerebrovascular disease, hypertension and hypercholesterolaemia was also significantly higher in the DFU group (all $p$ values $<0.001$ ) (Table 2).

All-cause mortality During a total of 81,116 person-years of follow-up, there were 3,619 deaths from any cause. DFU was associated with an increased risk of all-cause mortality (pooled RR, 1.89 [95\% CI 1.60, 2.23]) (Fig. 2). Crude event rates for all-cause mortality were 99.9 per 1,000 personyears in the DFU population and 41.6 in the diabetes-only population. There was evidence of statistical heterogeneity in the effect estimate among studies $\left(I^{2}=64.2 \%, p=0.01\right)$.

In subgroup analysis stratified according to duration of follow-up, we did not observe any material differences in RR between studies reporting mean duration of follow-up as $\leq 3$ and $>3$ years $(2.01$ [95\% CI 1.85, 2.19] and 1.62 [95\% CI $1.40,1.88]$, respectively). There was greater heterogeneity between studies with a mean follow-up duration of $>3$ years compared with studies reporting $\leq 3$ years follow-up ( $I^{2}=69.8 \%, p=0.02$ and $I^{2}=10.4 \%, p=0.34$, respectively) (Table 3). Funnel plot inspection (Fig. 3), Egger's test $(p=0.95)$ and Begg's test $(p=0.90)$ demonstrated no significant publication bias among studies included in this meta-analysis.

Fatal CVA and MI Information on cardiovascular mortality was available for a total of 3,138 patients across four studies. There were 113 deaths from CVA and 359 deaths from MI during 28,189 person-years of follow-up. Cardiovascular deaths accounted for 51 of $117(43.6 \%)$ deaths in patients with DFU and 421 of 952 (44.2\%) deaths in patients with diabetes only. The RRs for MI and CVA were 2.22 (95\% CI $1.09,4.53)$ and $1.41(95 \%$ CI $0.61,3.24)$, respectively. There was no evidence of significant heterogeneity between studies for these outcomes (fatal MI $I^{2}=48.1 \%, p=0.12$; fatal CVA $\left.I^{2}=16.2 \%, p=0.31\right)$. Crude event rates per 1,000 person-years were 16.8 and 11.8 for fatal MI in the DFU and diabetes-only populations, respectively; 4.6 and 3.8 fatal
CVAs occurred per 1,000 person-years in the DFU and diabetes-only groups, respectively.

\section{Discussion}

We provide the largest scale evidence to date that patients with DFU have a higher risk of all-cause mortality compared with patients with diabetes but without a history of foot ulceration. We observed an association for increased risk of fatal MI and a trend towards an increased risk of CVA in patients with DFU, although data on combined incident CVD deaths were not consistently available across the studies included.

While the number of CVD deaths is greater in a DFU population, our data demonstrate that equal proportions of overall deaths in both DFU and diabetes-only patients are attributable to cardiovascular causes. In studies reporting on cardiovascular and all-cause mortality, fatal MI and CVA accounted for $44 \%$ of total deaths in both DFU and diabetesonly groups. These findings imply that the excess CVD risk observed in DFU patients only partly accounts for the increased mortality rate. Excess mortality in patients with DFU may equally reflect a more advanced stage of diabetes with greater disease burden and non-cardiovascular complications of foot ulceration such as sepsis. Our data support the hypothesis that, in order to attenuate the excess mortality associated with DFU, strategies focusing on both more aggressive modification of CVD risk factors and ulcer prevention might be required.

Potential explanations for the association of DFU and mortality Evidence suggests that mortality is higher with ischaemic ulcers than with neuropathic ulcers [16]. Accordingly, peripheral arterial disease (PAD) is a consistent independent predictor of CVD and all-cause mortality. In addition to the association of macrovascular disease and mortality, a complex interplay of factors related to both sequelae of ulceration and the longer duration of disease
Table 2 Comparisons of baseline demographics and cardiovascular risk factors

Values are presented as means or numbers $(\%)$

${ }^{\mathrm{a}} \chi^{2}$ compared categorical variables; continuous variables were compared using independent $t$ tests

\begin{tabular}{llll}
\hline Characteristics & DFU & Diabetes only & $p$ value $^{\mathrm{a}}$ \\
\hline Age (years) & 63.7 & 66.2 & $<0.005$ \\
Time since diagnosis of diabetes (years) & 12.7 & 7.2 & $<0.001$ \\
Type 2 diabetes & $273 / 330(82.7)$ & $1,379 / 1,637(84.2)$ & 0.494 \\
Men & $2,542 / 3,007(84.5)$ & $8,198 / 14,098(58.2)$ & $<0.001$ \\
CAD & $921 / 2,934(31.4)$ & $2,041 / 13,923(14.7)$ & $<0.001$ \\
CVA & $411 / 2,934(14.0)$ & $1,131 / 13,923(8.1)$ & $<0.001$ \\
Current smokers & $66 / 320(20.6)$ & $(497 / 2,918) 17.0$ & 0.107 \\
Hypertension & $480 / 834(57.6)$ & $4,223 / 11,823(35.7)$ & $<0.001$ \\
Hypercholesterolaemia & $30 / 63(47.6)$ & $162 / 1,456(11.1)$ & $<0.001$ \\
\hline
\end{tabular}




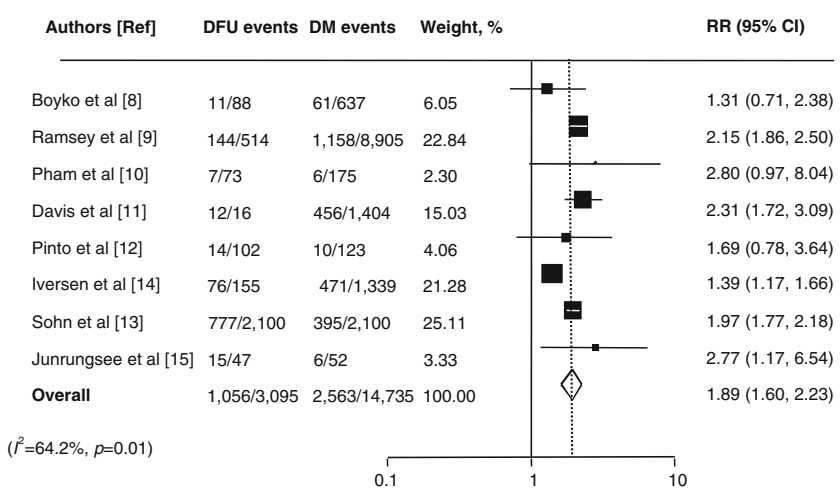

Fig. 2 RR for all-cause mortality by DFU vs diabetes only. $I^{2}=64.2 \%$; $p=0.01$

seen in patients with DFU are likely to contribute to the observed independent association of DFU and all-cause mortality. Active ulceration has been shown to elicit numerous adverse biological responses, most notably chronic inflammation, which has an established role in the development and progression of atherosclerosis.

Peripheral neuropathy is present in a significant proportion of patients at the time of diagnosis of type 2 diabetes [17], and progresses with duration of disease. Cardiovascular autonomic neuropathy $(\mathrm{CAN})$ is an often-overlooked complication of diabetes that frequently co-exists with distal polyneuropathy, microangiopathy and macroangiopathy, all important risk factors for the development of DFU. CAN is associated with increased mortality and silent myocardial ischaemia, a pooled estimate of RR derived from 15 studies involving 2,900 participants was 2.14 (95\% CI 2.66, 4.47, $p<0.0001)$ [18]. Somatic neuropathy has been implicated in the development of medial arterial calcification; indeed, it has been suggested that neuropathy explains the distal distribution of arterial calcification in individuals with diabetes [19].

We observed a greater heterogeneity of effect size between studies reporting a longer duration of follow-up, which may reflect a variable burden of disease seen in individuals with a similar duration of diabetes. A significant determinant of mortality in patients with diabetes at an advanced stage is the increased presence of renal disease, which is itself strongly linked with accelerated CVD and mortality. Diabetic nephropathy is the leading cause of renal failure in developed countries and an independent predictor

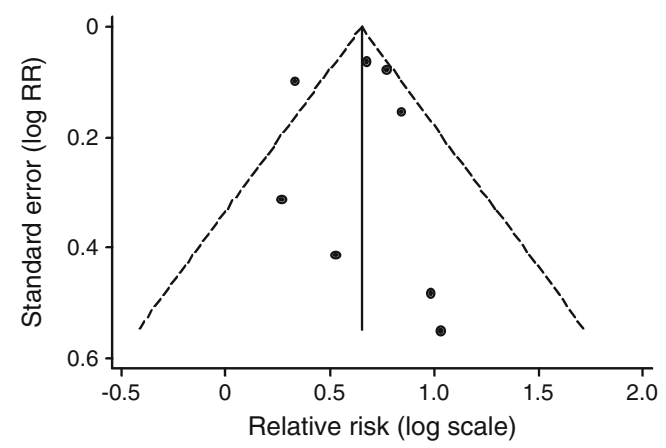

Fig. 3 Funnel-plot analysis, with pseudo 95\% CIs for all included studies

of all-cause and CVD mortality. In a report from the Early Treatment Diabetic Retinopathy Study, high serum creatinine among patients with type 2 diabetes was associated with the highest hazard ratio for mortality $(2.46$ [95\% CI $1.83,3.32]$ ) of all the complications of diabetes [20].

Implications for clinical care Despite stratification of ulcer risk in primary care in patients with diabetes, the effectiveness of screening programmes in both reducing the risk of foot ulceration and mortality is unclear [21]. Foot examination focusing on the presence of peripheral neuropathy, PAD and abnormal foot anatomy can predict patients at high risk of developing DFU [22]; however, the evidence for an intervention strategy in these patients is not well defined.

The benefit of aggressive cardiovascular risk management in diabetes has been clearly demonstrated in the extension of the Steno-2 study [23]. Patients with type 2 diabetes and persistent microalbuminuria were randomly assigned to receive conventional therapy or intensive therapy incorporating tight glucose regulation and the use of renin-angiotensin system blockers, aspirin and statins. Intensive therapy corresponded to an absolute risk reduction for all-cause mortality of $20 \%$ after a mean follow-up of 13.3 years ( 7.8 years of multifactorial intervention and an additional 5.5 years of follow-up). If we extrapolate this risk reduction to the present study, multifactorial intervention would result in 20 fewer deaths per 1,000 years in a DFU population, potentially halving the excess risk in DFU populations. It is recognised that participants in the Steno-2 study were selected for the presence of microalbuminuria

Table 3 Subgroup analysis according to duration of follow-up

\begin{tabular}{|c|c|c|c|c|c|c|c|}
\hline \multirow[t]{2}{*}{ Mean follow-up } & \multicolumn{2}{|l|}{$n$} & \multirow[t]{2}{*}{ Studies } & \multicolumn{2}{|c|}{ Number of events } & \multirow[t]{2}{*}{$\mathrm{RR}(95 \% \mathrm{CI})$} & \multirow[t]{2}{*}{$I^{2}$} \\
\hline & DFU & Diabetes only & & DFU group & Diabetes-only group & & \\
\hline$\leq 3$ years & 2,775 & 11,817 & 4 & $939 / 2,775$ & $1,620 / 11,817$ & $2.01(1.85,2.19)$ & 10.4 \\
\hline$>3$ years & 320 & 2,918 & 4 & $117 / 320$ & $943 / 2,918$ & $1.62(1.40,1.88)$ & 69.8 \\
\hline All & 3,095 & 14,735 & 8 & $1,056 / 3,095$ & $2,563 / 14,735$ & $1.89(1.60,2.23)$ & 64.2 \\
\hline
\end{tabular}


which is itself a strong predictor of cardiovascular events $[24,25]$. In addition, microalbuminuria is associated with microvascular disease and therefore neuropathy and ulceration.

In the setting of patients with DFU, Young et al reported improved survival of patients treated with aggressive cardiovascular risk management [26]. In a foot-clinic population, 5 year mortality fell from $48 \%$ to $27 \%$ following introduction of a protocol incorporating CVD-risk-factor screening and modification with an antiplatelet agent, a statin and an ACE inhibitor $\pm \beta$-blocker in those with hypertension.

Strengths and limitations As with all meta-analyses, there are limitations that are, in part, related to the data available. In particular, the studies included are observational reports and therefore we cannot exclude the potential impact of confounding. Specifically for the present study the duration of diabetes was inconsistently reported across included studies; where this information was available, the mean duration of disease varied from 6 to 16 years. Patients with a longer duration of disease may have a greater burden of diabetes complications, and potential confounding of mortality is possible. Patient populations also differed with respect to the duration of follow-up and the prevalence of CVD risk factors. Subgroup analysis showed that follow-up duration did not make a substantial difference in all-cause mortality, however; greater heterogeneity was observed in studies reporting a mean follow-up duration $>3$ years. The impact of confounding could have been reduced via the availability of individual participant data or adjusted tabular data to establish the impact of CVD risk factors on all-cause mortality. This was only available in two out of the eight studies despite our best efforts to supplement published findings with additional information. We do hope that the present findings encourage the collaborative, systematic and detailed use of published and unpublished individual participant data from cohorts around the world to examine with greater precision the associations reported here.

Although our study has over 80,000 person-years of follow-up, the studies included reported on a total of only 472 incident CVD deaths; more data on the cause-specific mortality would have provided insights into the relative influence of ulcer-related factors on mortality in DFU. Sufficient data on the timing of ulceration and subsequent events were lacking and we were therefore unable to produce hazard ratios to evaluate the relationship between active ulceration and mortality.

Implications for future studies Within the limitations of the present study design our findings suggest that patients with diabetes and ulceration of the foot may be at considerably higher risk of all-cause mortality compared with those patients with diabetes but no history of foot ulceration. This may in part be explained by a greater burden of CVD risk factors and subsequent excess risk of fatal cardiovascular events. Further investigation is warranted to clarify the relative roles of ulcer sequelae and CVD to inform strategies that may further attenuate the significant morbidity and mortality in this group of patients. Randomised trials are needed to address whether greater control of risk factors or screening impact on causespecific mortality.

Funding This study was not funded by any external sources, and JRWB, JD, KKR and RJH take full responsibility for the data presented and the decision to submit for publication.

Duality of interest The authors declare that there is no duality of interest associated with this manuscript. All authors have completed the Unified Competing Interest form at www.icmje.org/coi_disclosure.pdf. All authors declare: no support from any organisation for the submitted work; no financial relationships with any organisations that might have an interest in the submitted work in the previous 3 years; and no other relationships or activities that could appear to have influenced the submitted work.

Contribution statement $\mathrm{KKR}$ and RJH contributed equally to this work. KKR, RJH and MMT were responsible for the conception and design of the study. JRWB, PJH and WAD were responsible for the acquisition of data. JRWB, PJH and JD performed the analysis. All authors critically revised the manuscript for important intellectual content and approved the final version of the manuscript.

\section{References}

1. Preis SR, Hwang SJ, Coady S et al (2009) Trends in all-cause and cardiovascular disease mortality among women and men with and without diabetes mellitus in the Framingham Heart Study, 1950 to 2005. Circulation 119:1728-1735

2. Pham H, Armstrong DG, Harvey C, Harlkess LB, Giurni JM, Veves A (2000) Screening techniques to identify people at high risk for diabetic foot ulceration. Diabetes Care 23:606-611

3. Silbernagel G, Rosinger S, Grammer TB et al (2012) Duration of type 2 diabetes strongly predicts all-cause and cardiovascular mortality in people referred for coronary angiography. Atherosclerosis 221:551-557

4. Wannamethee SG, Shaper AG, Whincup PH, Lennon L, Sattar N (2011) Impact of diabetes on cardiovascular disease risk and allcause mortality in older men: influence of age at onset, diabetes duration, and established and novel risk factors. Arch Intern Med 171:404-410

5. DerSimonian R, Laird N (1986) Meta-analysis in clinical trials. Control Clin Trials 7:177-188

6. Begg CB, Mazumdar M (1994) Operating characteristics of a rank correlation test for publication bias. Biometrics 50:1088-1101

7. Egger M, Davey Smith G, Schneider M, Minder C (1997) Bias in meta-analysis detected by a simple, graphical test. BMJ 315:629634 
8. Boyko EJ, Ahroni JH, Smith DG, Davignon D (1996) Increased mortality associated with diabetic foot ulcer. Diabet Med 13:967-972

9. Ramsey SD, Newton K, Blough D et al (1999) Incidence, outcomes, and cost of foot ulcers in patients with diabetes. Diabetes Care 22:382-387

10. Pham H, Armstrong DG, Harvey C, Harkless LB, Giurni JM, Veves A (2000) Screening techniques to identify people at high risk for diabetic foot ulceration. Diabetes Care 23:606-611

11. Davis WA, Norman PE, Bruce DG, Davis TME (2006) Predictors, consequences and costs of diabetes-related lower extremity amputation complicating type 2 diabetes: The Fremantle Diabetes Study. Diabetologia 49:2634-2641

12. Pinto A, Tuttolomondo A, Di Raimondo D et al (2008) Cardiovascular risk profile and morbidity in subjects affected by type 2 diabetes mellitus with and without diabetic foot. Metabolism 57:676-682

13. Sohn MW, Todd AL, Stuck RM, Frykberg RG, Budima-Mak E (2009) Mortality of charcot arthropathy compared with that of diabetic foot ulcer and diabetes alone. Diabetes Care 32:816-821

14. Iversen MM, Tell GS, Riise T et al (2009) History of foot ulcer increases mortality among individuals with diabetes. Ten-year follow up of the Nord-Trondelag Health Study, Norway. Diabetes Care 32:2193-2199

15. Junrungsee S, Kosachunhanun N, Wongthanee A, Rerkasem K (2011) History of foot ulcers increases mortality among patients with diabetes in Northern Thailand. Diabet Med 28:608-611

16. Moulik PK, Mtonga R, Gill GV (2003) Amputation and mortality in new-onset diabetic foot ulcers stratified by etiology. Diabetes Care 26:491-494

17. UKPDS 33 (1998) Intensive blood glucose control with sulphonylurea or insulin compared with conventional treatment and the risk of complications in type 2 diabetes. Lancet 352:837-853
18. Vinik AI, Ziegler D (2007) Diabetic cardiovascular autonomic neuropathy. Circulation 115:387-397

19. Jeffcoate WJ, Rasmussen LM, Hofbauer LC, Game FL (2009) Medial arterial calcification in diabetes and its relationship to neuropathy. Diabetologia 52:2478-2488

20. Cusick M, Meleth AD, Agron E et al (2005) Associations of mortality and diabetes complications in patients with Type 1 and Type 2 diabetes. Early treatment diabetic retinopathy study report no 27. Diabetes Care 28:617-625

21. Jeffcoate WJ (2011) Stratification of foot risk predicts the incidence of new foot disease, but do we yet know that the adoption of routine screening reduces it? Diabetologia 54:991993

22. Abbott CA, Carrington AL, Ashe $\mathrm{H}$ et al (2002) The North-West Diabetes Foot Care Study: incidence of, and risk factors for, new diabetic foot ulceration in a community-based patient cohort. Diabet Med 19:377-384

23. Gaede P, Lund-Andersen H, Parving HH, Pedersen O (2008) Effect of multifactorial intervention on mortality in type 2 diabetics. N Engl J Med 358:580-591

24. Dinneen SF, Gerstein HC (1997) The association of microalbuminuria and mortality in non-insulin dependent diabetes mellitus. Arch Intern Med 157:1413-1418

25. Agewall S, Wikstrand J, Ljungman S, Fagerberg B, for the Risk Factor Intervention Study Group (1997) Usefulness of microalbuminuria in predicting cardiovascular mortality in treated hypertensive men with and without diabetes mellitus. Am J Cardiol 80:164-169

26. Young MJ, McCardle JE, Randall LE, Barclay JI (2008) Improved survival of diabetic foot ulcer patients 1995-2008: possible impact of aggressive cardiovascular risk management. Diabetes Care $31: 2143-2147$ 